\title{
Il teatro delle piccole cose: Quiet Ensemble
}

\author{
Maia Giacobbe Borelli
}

\begin{abstract}
II mio contributo partecipa alla mappatura delle pratiche performative italiane. Riprendendo quanto introdotto da Auslander con Liveness nel 1999, alla luce di recenti riflessioni e di sperimentazioni artistiche e mediali in contesti offline e online, il saggio esplora il lavoro di Quiet Ensemble, factory crossmediale composta dagli artisti-performers Fabio Di Salvo e Bernardo Vercelli. Le loro performance consentono di introdurre maggiori gradi di complessità al concetto di liveness. II duo opera dal 2009 al confine tra arte, video, teatro e musica digitale, presentando spettacoli e installazioni fortemente tecnologiche, realizzate con l'utilizzo di elementi del mondo naturale (luce solare, topi, api, pesci, ecc.) che sono proposti dal vivo, in un percorso predeterminato, e che con il loro movimento realizzano essi stessi l'opera in modo aleatorio, nel quadro che la tecnologia digitale preparata da Quiet Ensemble mette loro a disposizione. Gli artisti-performer interagiscono in scena con la situazione che si viene a creare grazie alla presenza di questi insoliti protagonisti.
\end{abstract}

This essay explores the work of Quiet Ensemble, a cross-media factory by artist-performers Fabio Di Salvo and Bernardo Vercelli. My analysis begins from the concept of Liveness (Auslander, 1999) as it relates to recent reflections on art and media practices in on and off-line environments and is intended to contribute to a mapping of new Italian performance practices. In my opinion Quiet Ensemble's performances opens various degrees of complexity that further expand the concept of Liveness. Di Salvo and Vercelli have created live performances since 2009. Their work is located at the intersection of art, video, theater and digital music. They present highly technological shows and installations created with "elements" from the natural world - such as sunlight, mice, bees, fish and more. The performances are created by the random interaction of these "elements" with the digital framework controlled live, on stage, by Di Salvo and Vercelli.

\section{Parole chiave/Key Words}

Parole chiave; liveness, natura, organicità, crossmedialità, performance digitale offline.

Key words; liveness, nature, organic, offline performance, crossmedia. 
Da metamorfosi indescrivibile sgorgano tali forme - senti ! e credi ! Noi soffriamo sovente : le fiamme son fatte cenere, ma, nell'arte, la polvere diviene fiamma.

Qui è la magia. Nel regno del magico la parola comune è istituita ma è reale come il richiamo del colombo che chiama l'invisibile colomba.

Rainer Maria Rilke, Magie

Ci occuperemo qui di una singolare esperienza artistico-performativa, Quiet Ensemble, factory crossmediale a dimensione altamente tecnologica. II duo di artisti, che rispondono al nome di Bernardo Vercelli e Fabio Di Salvo, dal 2009 gira l'Europa, gravato da un carico notevole di hardware e software per la produzione sonora e visiva: superfici connesse dove si muovono piccoli animali oppure riflettori di ultima generazione che agiscono come un'orchestra di suoni. Una ricerca sul linguaggio della luce o del movimento che crea eventi utilizzando la materia vivente $^{1}$. Il lavoro è incentrato sulla creazione di installazioni che sono anche spazi performativi, e che esplorano i fenomeni naturali e i meccanismi di percezione, resi evidenti attraverso l'azione sullo spazio e la luce ispirata da modelli atmosferici e da forme di biomeccanica naturale.

Da un approccio sostanzialmente artistico e ludico, la factory è approdata a una dimensione che ha, a mio avviso, una forte impronta performativa. Non si tratta però di registrare un passaggio transdisciplinare che può sembrare fine a se stesso, quanto di verificare un paradigma che sempre più spesso ci porta a confondere i limiti disciplinari tra le arti, lasciandoci incapaci di stabilire se quanto offerto alla fruizione del pubblico appartenga ancora al dominio del teatro o sia ormai sconfinato nel territorio vasto e indistinto delle arti visive e sonore. Essenziale alla comprensione della novità epistemologica è un'iniziale riflessione sulle coordinate della ricerca qui proposta all'attenzione, per la quale è necessaria una rimessa a regime del concetto di liveness alla luce di questa sperimentazione artistica e mediale. Le opere proposte dall'ensemble sono varie, secondo differenti contesti d'uso e tecnologie impiegate, in relazione agli spazi utilizzati e alle tematiche prescelte per esplorare il rapporto

\footnotetext{
1 I loro progetti artistici sono stati presentati varie volte al RomaEuropa Festival, al MAXXI di Roma e alla Triennale di Milano, oltre che apprezzati in sede internazionale, al Festival Sonar di Barcellona, come a W.I.R.E., a Hellerau, al TransArt, al DMY, tra gli altri. Fra i software utilizzati da Quiet Ensemble: Max msp, Mad mapper, Ableton live; Processing, Videomapping e Hardware: Arduino, Raspberry P.
} 
tra organicità e mondo artificiale, che è la loro principale ossessione. La tecnologia digitale è strumento necessario e indispensabile per avvicinarsi alla contemplazione degli elementi organici e delle più minute cose terrestri. I dispositivi di tracciamento visivo e sonoro permettono di amplificare la micro vitalità della materia proposta all'attenzione (volta per volta non umana, animale, inanimata) e di concentrare l'attenzione dello spettatore sulle "piccole cose".

\section{Mettere in scena le piccole cose}

Al centro delle performance-installazioni proposte troviamo sempre il mondo naturale, messo in scena in varie forme: dall'osservazione del movimento di piccoli animali alla rappresentazione accelerata degli effetti del vento o del mutamento della luce solare in un particolare ambiente chiuso che funge da spazio scenico, come il Salone degli Incanti di Trieste nell'azione-installazione Sole del 2021.

Bernardo Vercelli sostiene di aver iniziato il proprio itinerario artistico collaborando con gli animali, nello specifico con alcuni pesci rossi, «che abbiamo fatto suonare, attribuendo loro degli strumenti musicali digitali che producevano suono con il loro movimento ${ }^{2}$. Nell'installazione Quintetto ${ }^{3}$ i pesci rossi animano la luminosità e producono il suono percepito. Solo lo spettatore attento riesce ad avvertire il legame tra il movimento dei pesci e la dinamica di suono e luce di cui si compone l'opera.

Numerose sono le proposte artistiche del duo sviluppate nel corso degli anni: nel percorso storico del gruppo, ai pesciolini musicisti segue il progetto Allegoria ${ }^{4}$, con sensori mioelettrici applicati alle braccia di musicisti di un quartetto d'archi, che permettevano di

\footnotetext{
${ }^{2}$ B. Vercelli, intervista del 7 settembre 2021. Vercelli, di formazione scenografo, ha frequentato la Akademi fur Scenekunst in Norvegia, dove ha conosciuto l'opera immersiva del danese Olafur Eliasson e degli olandesi dello Studio Roosegaarde, ai quali si ispira nel lavoro. Per maggiori informazioni sull'opera di Eliasson vedi sitografia e l'intervista rilasciata nel 2015 da Eliasson al sito: www.designboom.com/art/olafur-eliasson-interview-artist-designboom-02-16-2015 (ultimo accesso 28/11/2021). Per lo Studio Roosegaarde vedi info a www.studioroosegaarde.net/stories (ultimo accesso 28/11/2021).

${ }^{3}$ Quintetto, 2009, presentato a Tonlagen (Hellerau, Europäisches Zentrum der Künste, Dresda, Germania), Semibreve Festival (Theatro Circo, Braga, Portogallo) Heart Of Noise Festival (Gotiskerkeller, Innstbruck, Austria), Sound Lectures, (Superbudda, Circolo dei lettori, Torino), Biennale Panoramas (Le parc des Coteaux, Bordeaux, FR), Oddstream Festival (Lindenberg, Nijmegen, Paesi Bassi), Interstice Festival (Quai François Mitterrand, Caen, Francia), Digital Life / Romaeuropa (Ex Gill, Roma), DMY - International Design Festival (Tempelhof Airport, Berlino, Germania), Premio Celeste (primo premio) (Fondazione Brodbeck, Catania), Short Theatre (Pelanda MACRO, Roma), Drodesera (Centrale Fies Drò, Trento). I pesciolini rossi erano gli unici viventi in scena in questa prima performance cui i due artisti sono molto legati.

${ }^{4}$ Allegoria, 2012: in collaborazione con The String Quartet, Quartetto Berio (Teatro Valle, Roma), Quit Festival (Ghetto Ebraico, Cagliari), Nuit Blanche Bruxelles (Chapelle de Nassaukapel, Bruxelles, Belgio), Re_New Festival (PB43, Copenaghen, Danimarca).
} 
percepire un'altra musica, quella dei muscoli in movimento, insieme a quella dei violini. Poi l'installazione Orienta, è qui ora che ho deciso di fermarmi ${ }^{5}$, dove alcune lumache lentamente disegnano tracciati luminosi grazie al loro passaggio su una superficie sensibile. A fine giornata, queste lasciano un disegno imprevedibile fatto di luce, a testimonianza del loro percorso, disegno che rappresenta solo una porzione, della durata di poche ore, della loro stessa esistenza. Avvicinamenti e allontanamenti, traiettorie che sembrano frutto di decisioni, da cui il titolo dell’opera. Così Vercelli spiega la poetica della factory:

Siamo andati avanti con gli animali e abbiamo fatto collaborazioni con vermi, mosche, uccellini, topi poi abbiamo iniziato anche a lavorare con gli elementi naturali: il vento e la gravità con Unshaped ${ }^{6}$, progetto che sta avendo una nuova vita adesso dopo un debutto che risale a qualche anno fa. ${ }^{7}$

Questo progetto invece vede in scena una grande tela mossa da ventilatori, e suggerisce secondo il contesto in cui viene inserita un'aurora boreale, come a Helsinki, oppure il respiro di un enorme essere, il movimento e il suono del mare, come a Palermo all'Oratorio di San Mercurio:

Unshaped è una installazione organica, in continuo movimento: si mostra al pubblico alle volte come un paesaggio sottomarino, il cielo, un'onda e poi ancora una nuvola, acqua e aria, leggera o pesante. Un'orchestra di luci fluttua nello spazio trasformando gli spazi dell'Oratorio di San Mercurio, con le sculture del Serpotta, gli stucchi abbaglianti, le cornici barocche e il pavimento maiolicato, in una sorta di acquario in cui danzano acqua e aria, tra riflessi e ombre, moltiplicandosi e scomparendo, in un'eclissi frammentata e stroboscopica. ${ }^{8}$

Con Unshaped, la drammaturgia di luci e suoni di ventilatori compone una visione in chiave trascendentale e mistica del "senza forma" naturale, fatto di aria e luce. Vercelli riprende il filo delle opere realizzate, in una veloce cronologia, spiegando un'altra opera, Enlightenment:

\footnotetext{
${ }^{5}$ Orienta, 2012: RomaEuropa, Digital Life (Macro, Roma), Recto Verso, MOIS Multi Festival (Quebec City, Canada), Transart Festival (Galleria Civica, Bolzano).

6 Unshaped, 2016-2022. presentato a: Llum Bcn (MUHBA Oliva Artés, Barcellona, Spagna), Signal Festival (Hauch Gallery, Praga, Repubblica Ceca), Electric Castle (Cluj Napoca, Romania), Wall Of Sounds (Oratorio San. Mercurio, Palermo), RoBOt Festival (Ex GAM, Bologna), Outdoor Festival al MAXXI (Ex Caserma Guido Reni, Roma) e a Lux Helsinki (Finlandia) nel gennaio 2022.

${ }^{7}$ B. Vercelli, op. cit.

${ }^{8}$ M. Manicardi, I/ Barocco immersivo di Quiet Ensemble, all'Oratorio S. Mercurio di Palermo, Exibart, recensione del 29 dicembre 2020, disponibile a: https://www.exibart.com/progetti-e-iniziative/unshaped-quiet-ensemblepalermo (ultimo accesso 28/11/2021).
} 
Abbiamo iniziato ad analizzare il mondo microscopico, l'invisibile e inudibile, mostrando questi concerti o teatri nascosti, che abbiamo sempre sotto i nostri occhi, ma di cui non ci rendiamo conto. Da lì abbiamo iniziato ad analizzare anche la vita e l'anima delle cose, degli oggetti, che hanno anch'essi un processo d'invecchiamento indipendente dall'attività umana. Abbiamo iniziato a lavorare con le frequenze elettriche di fari teatrali e la ricerca è diventata quella che è apparsa con il progetto Enlightenment ${ }^{9}$, dove abbiamo creato un concerto che usa il suono dei fari teatrali, nello specifico la "ronza", così chiamiamo quei ronzii prodotti dall'elettricità che di solito sono nascosti e per noi diventano invece lo strumento musicale. Abbiamo messo delle bobine di rame a ogni faro, creando una vera e propria orchestra composta di 96 elementi luminosi, ognuno microfonato e amplificato. E a fine spettacolo, i fari accecavano il pubblico dando loro una percezione fisica di calore. ${ }^{10}$

Si tratta, come spiega Olafur Eliasson, di considerare le opere come "produttori di fenomeni", meccanismi immersivi e dispositivi scenici che lavorano con la luce, con il suono e con lo spazio producendo un certo evento in modo più o meno illusorio e sempre adeguato al luogo in cui sono collocati. II punto centrale è poi quello di rivelare l'illusione, di non nasconderla, e di indurre lo spettatore a mettere in campo la propria soggettività, prendendo posizione non di fronte all'opera ma proprio all'interno di essa ${ }^{11}$. Conclude Vercelli:

\begin{abstract}
Abbiamo ripreso la strada precedente con l'ultimo progetto, Sole ${ }^{12}$, con il quale stiamo ricreando la natura attraverso la tecnologia, dove questa è invisibile e anzi ti spiazza. II pubblico entrante al Salone degli Incanti, a Trieste, non percepisce neanche la presenza di un'installazione se non nel momento in cui la luce del sole va più veloce, o sorge un altro sole nella parte sinistra della sala. In questo caso abbiamo poi ricreato il progredire del sole nell'arco di una giornata. Siamo passati dalle formiche al sole, questo per ora è il nostro punto di arrivo. ${ }^{13}$
\end{abstract}

\title{
Un'arte effimera e inafferrabile
}

Fabio Di Salvo sottolinea la componente effimera della loro proposta. Se riprodotta sempre nello stesso modo, l'opera si formalizza e perde d'intensità, per questo chiarisce che non si sono mai interessati a esperienze che mettano al centro della sperimentazio-

\footnotetext{
${ }^{9}$ The Enlightenment, 2014, Recto Verso - Mois Multi Festival (Quèbec city, Canada), Eufonic Festival (Madrid, Spagna), L'arsenale Festival (Museo Santa Caterina, Treviso), Eufònic FESTIVAL (Sant Carles de la Ràpita, Spagna), Sonar+D (Madrid, Spagna), Mirage Festival (Les Subsistances, Lione, Francia), Novasfrequencias Festival Oi Futuro Ipanema (Rio De Janeiro, Brasile), Romaeuropa Festival, Macro Testaccio (La Pelanda, Roma), Heart Of Noise Festival (Gotiskerkeller, Innstbruck, Austria), roBOt Festival (Palazzo Re Enzo, Bologna), Sere d'estate a Castel Sant'Angelo (Roma).

${ }^{10}$ B. Vercelli, op. cit.

${ }^{11}$ Cfr. O. Eliasson, Conversation entre Daniel Burnbaum et Olafur Eliasson, in M. Grynstejn, D. Birnbaum, M. Speaks (a cura di), Olafur Eliasson, Phaidon, London 2008, pp. 6-33.

12 Sole, 2021, Salone degli Incanti, Trieste.

${ }^{13}$ B. Vercelli, op. cit.
} 
ne performativa la realtà aumentata, che è spesso preregistrata. Se nel primo periodo della sperimentazione, per 5-6 anni, si sono concentrati sulla presenza in scena di esseri viventi fragili e sul loro movimento casuale, di cui il primo è stato quello randomico del pesce rosso, ultimamente, a causa del confinamento indotto dalla pandemia, il performer si è chiesto come continuare a produrre opere effimere facendole fruire in remoto al pubblico, senza ancora trovare il modo di proporre una simile azione all'interno della propria poetica. Come proporre diverse forme di esperienza in un ambiente regolato come quello virtuale? Riflette:

\begin{abstract}
L'annuncio di Zuckerberg sulla promozione della piattaforma di realtà virtuale Metaverse ${ }^{14}$ è interessante, perché è vero, a noi manca lo spazio giusto per progettare nuove forme artistiche, e anche se il suo l'obiettivo primario sembra essere prettamente commerciale, spostando l'interesse dei giovani dai social media al Metaverso, che sarà forse solo una riproposizione allargata di Second Life $^{15}$, speriamo resti uno spazio, anche se residuale, per la creazione artistica. lo penso a una situazione come quella del film di Spielberg «Ready Player One» ambientato nel 2045. ${ }^{16}$
\end{abstract}

L'uso "artistico" del Metaverso potrebbe essere possibile, a mio parere, riuscendo a mettere in una posizione critica il soggetto che partecipa, dandogli la capacità di vedere in modo diverso ed estraniante la sua stessa posizione nell'ambiente immersivo proposto. Un meccanismo di tipo brechtiano che non è facile ottenere.

Esiste una frontiera sottile e subliminale dove si confondono la nostra posizione reale e quella della rappresentazione e su questa frontiera sarebbe interessante agire, per risvegliare la coscienza dello spettatore sulla propria condizione nel mondo delle rappresentazioni del contemporaneo. Prosegue Di Salvo:

Sono convinto che riusciremo a trovare un modo di sfruttare questi linguaggi per portare avanti il nostro discorso, anche se la nostra forma di espressione migliore è l'effimero, che è un po' il contrario di quanto si possa fare online dove di tutto rimane la traccia. Con Orienta abbiamo provato a vendere le stampe risultanti dal percorso delle lumache. Forse oggi i NFT (Not Fungible Token), attribuendo un codice univoco all'oggetto digitale, possono permettere

\footnotetext{
${ }^{14}$ La parola sta per "Meta-universo": Metaverso è un termine coniato da Neal Stephenson in Snow Crash, libro di fantascienza cyberpunk, descritto come una sorta di realtà virtuale condivisa tramite internet, dove si è rappresentati in tre dimensioni attraverso il proprio avatar.

${ }^{15}$ Second Life è una piattaforma di realtà virtuale lanciata nel 2003 dalla società americana Linden Lab dove gli utenti potevano agire attraverso un avatar. Ha avuto quasi un milione di utenti negli anni migliori, fino al 2017, per poi velocemente declinare.

${ }^{16}$ S. Spielberg, Ready Player One, 2018. Il film è tratto dal romanzo Player One del 2010 scritto da Ernest Cline.
} 
la vendita di opere d'arte digitali. Sappiamo che il grande problema del digitale era la sua facile riproducibilità, ora potremmo dare nuovamente un'unicità a prodotti artistici creati durante la nostra performance, ma questa non sarà mai riproducibile. Già l'ultimo lavoro, Sole va più nella direzione della permanenza, data la grande quantità di materiale preregistrato, anche se l'attrezzatura tecnologica necessaria risulta sempre deteriorabile e la riporta dentro una connotazione effimera. Lavoriamo su una documentazione sempre migliore, nell'ottica di lasciare una traccia nel tempo, più che sul lasciare un'opera. Vedremo più avanti se nascerà in noi l'esigenza di prevedere qualcosa che resti nel tempo. Ma ci sarà sempre un discorso di fragilità dell'opera, sarà una sfida. ${ }^{17}$

Chiaro è che la produzione performativa di questa factory non potrebbe esistere senza i migliori software attualmente disponibili ${ }^{18}$. Infatti, l'uso estremo di dispositivi digitali nello spazio in cui agiscono costruisce l'unica drammaturgia possibile, quella scaturita dalla traccia sonora o luminosa del movimento aleatorio che l'animale vivente produce in scena. Perché veri protagonisti sono proprio i piccoli esseri, formiche, api, topolini, pesciolini, criceti, la cui presenza viva, nel tempo della performance, ci troviamo a osservare con un'attenzione insolita. Naturalmente, stabilire una serie di regole e di obiettivi predeterminati è condizione essenziale di tutti gli spettacoli e anche di questo, dove è necessario creare un contesto regolativo all'interno del quale l'imprevedibilità del movimento animale possa svilupparsi a pieno regime. E proprio nel conflitto tra la necessità di controllare l'azione messa in scena e l'impossibilità di prevedere i movimenti dei piccoli viventi si crea la tensione che mantiene viva l'azione drammatica dell'evento. Questa opposizione porta a intravedere una breve e sempre diversa narrazione, nonostante l'elemento aleatorio ne venga in ultima analisi esaltato. Oltre ai piccoli animali, però, non dimentichiamo che ci sono in scena i corpi dei due attori-performer che si dibattono attenti al comando dei macchinari. Vero è, come già affermato da Michael Kirby, che in questo tipo di rappresentazioni «the referential elements are applied to the performer and are not acted by him ${ }^{19}$. E infatti non $c^{\prime}$ è nessuno che reciti, per i due artisti si tratta di effettuare alcuni movimenti necessari, quello che potremmo chiamare task based performing, un agire scenico legato esclusivamente alla necessità di compiere un'azione non narrativa ma fattuale.

\footnotetext{
${ }^{17}$ F. Di Salvo, intervista del $1^{\circ}$ novembre 2021. Di Salvo si forma al DASS Sapienza e comincia un percorso legato ai dispositivi digitali, per unirsi nel 2009 a Vercelli per il progetto Quiet Ensemble.

${ }^{18}$ Solo l'opera Carillon ha escluso i dispositivi digitali, dato che alcuni criceti, girando su ruote, azionavano dei carilIon a cui queste erano collegate, producendo tutti insieme una musica aleatoria di tipo meccanico e non digitale.

${ }^{19}$ M. Kirby, On acting and not acting, in G. Battcock e R. Nickas (a cura di), The Art of Performance, Dutton, New York 1984, p. 100.
} 


\section{Esplorare la relazione tra la natura e i dispositivi di visione}

Noi spettatori ci troviamo a distillare storie che sono create dai percorsi spaziali e sonori di questi insoliti "attori", traiettorie di punti luminosi ed echi, percepibili grazie ai più recenti software e hardware; questi sono indispensabili al lavoro di Quiet Ensemble, ma costituiscono esclusivamente mezzi per focalizzarsi su quello che è al di là dell'ambiente virtuale creato dall'uomo, nella natura stessa. E di conseguenza, i dispositivi mediatici consentono un'attenzione al mondo naturale che, paradossalmente, è impossibile realizzare senza il loro intervento, anche se rappresentano quanto di più artificiale esista. D'altra parte, precisa già Auslander nel noto studio sulla liveness, «live performance now often incorporates mediatization such that the live event itself is a product of media technologies ${ }^{20}$. Ne consegue che ogni spettacolo dal vivo è oggi prodotto attraverso dispositivi mediatici o si relaziona fortemente con esso, perfino quando ne nega in modo palese I'utilizzo ${ }^{21}$. Cosciente che sul rapporto tra visione, materia, non umano, ecologie, tecnologie è in corso un enorme dibattito, che non è il caso qui di riportare ${ }^{22}$, mi limito a constatare che già nel 1995 la Haraway riscontrava come "La visione è sempre questione del potere di vedere e forse della violenza implicita nelle nostre pratiche di visualizzazione. [...] La visione richiede strumenti di visione: un'ottica è una politica di posizionamento. Gli strumenti della visione mediano i punti di vista; non c'è visione immediata dai punti di vista dei soggiogati ${ }^{23}$. Da quest'affermazione emerge la necessità non di negare i dispositivi della visione quanto di posizionare diversamente il soggetto spettatore, pratica essenziale per fondare nuovi progetti conoscitivi, in questo caso riguardante esseri viventi non umani e fenomeni naturali, perché «noi non siamo i responsabili del mondo. Ci abitiamo solo, e cerchiamo di avviare conversazioni non innocenti attraverso i nostri strumenti prostetici, incluse le tecnologie della visualizzazione ${ }^{24}$.

\footnotetext{
${ }^{20}$ P. Auslander, Liveness, Performance in a mediatized culture, Routledge London \& New York 1999, p. 24.

${ }^{21}$ Come J.D. Bolter e R. Grusin insegnano, lo spettacolo si va via via modificando per assorbire il contesto mediatico da cui scaturisce, in una perenne remediation.

${ }^{22}$ Rimando a questo proposito alle opere di Judith Butler, Donna J. Haraway e Karen Barad, come alla panoramica degli studi proposta da Ilenia Caleo sull'argomento. Cfr. I. Caleo, Performance, materia, affetti, Una cartografia femminista, Bulzoni, Roma 2021.

${ }^{23}$ D. Haraway, Manifesto cyborg. Donne, tecnologie e biopolitiche del corpo, Feltrinelli, Milano 1995, pp. 116-117.

${ }^{24}$ Ivi, p. 126.
} 
Vorrei inoltre mettere l'accento sull'indeterminatezza dell'azione proposta da questo ensemble, scaturita proprio dalla scelta di impiegare in qualità di performer principalmente la materia organica e vivente. L'imprevedibilità, caratteristica che sfugge a regole costitutive convenzionali e alla loro ripetizione evento per evento, è una costante di questa ricerca, perché imponderabili e ingovernabili sono i percorsi degli animali introdotti nello spazio scenico e che, al di là del quadro rigido che li contiene, non si possono prevedere come invece è possibile fare con attori o attrici, grazie al tempo tradizionalmente consacrato alle prove ${ }^{25}$. Come nel caso di ogni attività performativa, le loro opere seguono una serie di principi: innanzitutto uno spazio-tempo circoscritto, che determina una realtà separata o comunque differente da quella ordinaria, all'interno del quale sono impiegate una pluralità di mezzi e tecniche, esibite e utilizzate in modo strumentale, allo scopo di raggiungere uno stato di contemplazione del mondo in generale (e della natura in particolare). Caratterizza il loro agire una relativa indipendenza formale dalle motivazioni e dagli scopi richiesti dal mercato dell'arte e del teatro e intriga la vertigine provocata nello spettatore dalla capacità dell'opera di trasfigurare la realtà, esibendo il vivente nelle sue forme più infime. La qualità aleatoria dell'azione proposta all'interno dell'opera (sia essa dovuta al movimento delle minuscole bestiole o alla simulazione del movimento del sole stesso), l'imprevedibilità dell'azione che si rivela durante la performance, diventa momento centrale dell'azione drammatica.

\section{Complessità del concetto di liveness}

La compresenza "fisica" nello spettacolo, ancor di più in periodo di pandemia, dove abbiamo vissuto le interdizioni di assembramento e la chiusura dei teatri, è diventata momento raro; ne consegue che un momento totalmente live diventa un importante momento di resistenza al discorso dominante, proprio perché permette quello scarto diventato oramai insolito nel quotidiano. Qual è il nucleo fondante del discorso che i due portano avanti? Al centro della ricerca c'è l'esplorazione di un possibile equilibrio tra na-

\footnotetext{
${ }^{25}$ Sulla drammaturgia indotta dai dispositivi digitali cfr. A. Pizzo, Neodrammatico digitale. Scena multimediale e racconto interattivo. Accademia University Press, Torino 2013.
} 
tura e tecnologia, che ha avuto negli anni varie ramificazioni operative. Una costante del lavoro d'ensemble è l'utilizzo delle strategie e della meraviglia del gioco.

Ora mi domando: l'azione all'interno delle performance di Quiet Ensemble è libera ed effimera, perché proposta dal vivo e sufficientemente imprevedibile, o è comunque frutto principalmente di una rielaborazione digitale che la preordina? Rivediamo brevemente il discorso di Auslander in merito a come intendere il carattere live di una performance: nel 1999 egli poneva un quesito essenziale per i suoi tempi, che era il seguente: "What is the status of live performance in a culture dominated by mass media?"26, concentrandosi sull'impatto dei mass media, in particolare della televisione, come spazi unici dello scambio culturale, a cui segue oggi un altro - altrettanto fondamentale - quesito: "Qual è lo status oggi della performance dal vivo in una cultura dominata dai social media?". Il contesto culturale di questi ultimi vent'anni ha notevolmente cambiato il panorama mediatico ribaltando il rapporto di produzione e fruizione dei mezzi di comunicazione, rendendo sempre più individuale l'approccio ai media grazie ai dispositivi personali. Già Baudrillard s'interrogava sui pericoli di un'unificazione degli spazi comunicativi, unificazione che rappresenta di fatto un contenimento degli spazi creativi disponibili alla sperimentazione ${ }^{27}$. Se questa individualizzazione della comunicazione è la realtà quotidiana di quasi ogni individuo sul pianeta ${ }^{28}$, quale importanza ha per la nostra riflessione uno spettacolo che si svolge dal vivo davanti a un piccolo pubblico, rispetto all'offerta di tanti altri momenti fruibili individualmente in remoto da un ben più grande pubblico? La risposta emerge quando consideriamo il concetto di liveness come possibilità che va modificandosi all'interno dei diversi contesti culturali e sociali. Infatti il concetto si trasforma insieme al mutare delle nostre disponibilità spazio-temporali quotidiane. Esso assume allora sfumature diverse oggi, grazie al prevalere di una distanza spazio-temporale in ogni

\footnotetext{
${ }^{26}$ Cfr. P. Auslander, 1999, op. cit., p. 1: «lt is absolutely clear that our current cultural formation is satutated with, and dominated by, mass media representations in general, and television in particular». Poiché la cultura oggi è veicolata quasi totalmente in ambiente virtuale, questo spazio sembra identificarsi come l'unico possibile per lo scambio tra individui. Televisione, radio, cinema, ormai classificate come old media, sono passate in secondo piano nella scala delle comunicazioni sociali, in favore dell'affermarsi di una serie di advanced information technologies che riportano il discorso socio-politico della realtà all'interno di un codice univoco che tutto racchiude, e questo codice è digitale ed è fruito da dispositivi individuali e unici, gli smartphone.

${ }^{27}$ Cfr. J. Baudrillard, Pour une critique de l'économie politique des signes, Gallimard, Parigi 1972.

${ }^{28} \mathrm{Si}$ intende qui che ormai quasi ogni individuo sul pianeta è entrato in contatto con la tecnologia digitale, utilizzando gli smartphone.
} 
tipo di comunicazione, incluso quella performativa. L'opera classica che si fruisce in diretta al cinema o in televisione ne è un esempio. In quest'accezione, possiamo concordare che il significato stesso di liveness applicato a uno spettacolo mediatizzato è soggetto a una serie di contingenze che non lo determinano in modo statico ma, al contrario, dinamico, ovvero che permettono variazioni di significato a secondo dei contesti d'uso ${ }^{29}$. In estrema sintesi potremmo dire che la presenza cosiddetta dal vivo è in fin dei conti un fenomeno prettamente mentale, sono io come soggetto che guarda a definirla: non tanto nel caso di un'incursione eccessiva dei media all'interno dello spettacolo dal vivo, quanto per l'assorbimento nella mia rappresentazione di un'epistemologia che deriva direttamente dai dispositivi mediatici impiegati nel quotidiano di ognuno ${ }^{30}$.

\section{La "disappearance" come sacca di resistenza}

Una cosa ancora persiste nell'ontologia dello spettacolo dal vivo, ed è la sua qualità di perenne disappearance, la sua scomparsa alla fine della rappresentazione stessa. Esso resta inafferrabile dal punto di vista della sua riproducibilità, perché la traccia che lascia, per esempio grazie alla sua registrazione audiovisiva, non corrisponde all'opera, anzi ne è, attraverso le necessarie repliche, una continua reinterpretazione, mai una copia conforme, al limite un frammento del processo. Di conseguenza la condizione essenziale della performance "dal vivo" rimane la disappearance, termine molto più efficace, a nostro parere, dell'italiano "fuggevolezza" o "scomparsa". Inoltre, e non è cosa da poco - e infatti lo possiamo constatare solo nei casi più riusciti - resta la volontà dello spettacolo teatrale di fare resistenza all'assimilazione culturale dominante ${ }^{31}$. Vorrei far notare che la proposta di mettere al centro della scena piccoli animali, quelli che spesso passano inosservati o sono considerati senza diritti e senza coscienza, porta lo spettatore a riflettere sulle infinite scale di valore che esistono nella società nel considerare la vita, sia degli umani sia dei non umani. Il discorso di Judith Butler sull'ordine biopolitico ci può essere utile a chiarire i termini che regolano oggi il diverso diritto alla vita e alla soggettività di tutti gli esseri viventi:

\footnotetext{
${ }^{29}$ Per esempio, in alcuni contesti lo spettacolo sarà vissuto come momento di scambio "dal vivo" anche se utilizza sequenze registrate e non opera all'interno di una condivisione spazio-temporale tra attori e spettatori.

${ }^{30}$ Cfr. P. Auslander, 1999, op. cit., p. 33.

${ }^{31}$ Cfr. P. Phelan, Unmarked: The Politics of Performance, Routledge, London \& New York, 1993.
} 
Nonostante io debba domandarmi come vivere una vita buona - e quest'aspirazione è importante -, devo pensare attentamente a questa vita che è la mia, ma che è anche una più ampia vita sociale, connessa con altri esseri viventi in modi che mi coinvolgono in una relazione critica con gli ordini discorsivi - sulla vita e il suo valore - in cui vivo o, piuttosto, in cui mi sforzo di vivere. Da dove traggono la loro autorità questi ordini? E tale autorità è legittima? Dato che anche la mia vita è in gioco in questa ricerca, la critica dell'ordine biopolitico è per me una questione vivente. ${ }^{32}$

Uno spettacolo che offre la scena a esseri normalmente considerati senza diritti, neanche quello della vita, è, in forma cosciente o inconscia - non sta a me deciderlo -, una forte critica all'ordine biopolitico. Quello che è interessante con Quiet Ensemble è che, con i codici tecnologici conosciuti dal pubblico, i performer costruiscono un discorso alternativo che si rivela, a volte, resistente al discorso dominante, specie quando la soggettività dello spettatore si riflette nel gioco sottile a cui lo conduce il progetto immersivo, dove sé e altro da sé perdono la loro qualità oppositiva razionale. Così come imprevedibile resta sempre e comunque la reazione dello spettatore di fronte all'azione proposta alla sua attenzione, la sua capacità o meno di trovare la quiete e di far emergere grazie ad essa il significato ultimo dell'evento proposto.

\section{L'arte della quiete}

Per cogliere la novità del progetto di questa factory, lo spettatore deve, infatti, fare suo l'invito alla quiete, cessare il proprio movimento o rallentarlo moltissimo, per costruire un suo proprio tempo di visione. E il rallentamento percettivo indotto nello spettatore mette in evidenza, per contrasto, l'artificialità della condizione umana. Fa resistenza. Possiamo riprendere le riflessioni relative alla condizione umana proposte dalla filosofa Hanna Arendt, quando spiega che l'opera assume la sua funzione proprio nel momento in cui esce dal contesto quotidiano per il quale è conosciuta. Per comprendere la necessità di creare uno spazio altro, nella performance "dal vivo", spazio separato dove condividere momenti lontani dalla vita quotidiana, riportiamo le sue parole:

Il giusto rapporto con un'opera d'arte non è certamente quella di "usarla"; essa al contrario deve essere allontanata accuratamente dall'intero contesto degli oggetti d'uso perché trovi

\footnotetext{
32 J. Butler, A chi spetta una buona vita?, Nottetempo, Milano 2013, pp. 16-17.
} 
il proprio posto nel mondo. Analogamente, deve essere anche sottratta alle esigenze e ai bisogni della vita quotidiana, con cui ha meno contatto che con ogni altra $\operatorname{cosa}^{33}$.

Prosegue la Arendt: «in realtà non c'è cosa che non trascenda in un modo o in un altro il proprio uso funzionale, e la sua trascendenza, la sua bellezza o bruttezza, corrisponde alla sua apparizione in pubblico e all'essere vista» ${ }^{34}$. Ovvero gli animali e il loro movimento sono quelli di sempre, ma metterli in scena li trascende, proprio perché c'è lo spettatore che li guarda con un'attenzione diversa. Anche secondo Olafur Eliasson l'obiettivo del lavoro è di proporre nello spettatore una possibilità di guardare diversamente il mondo, un "cambiamento di prospettiva" che fa dell'opera un vero e proprio catalizzatore $^{35}$. L'opera ha bisogno di trascendenza per diventare arte, di segnalare qualcosa di esterno che permette chi guarda di andare al di là. Ce lo spiega molto bene JeanLuc Nancy quando dice che «L'opera d'arte produce un segno che va al di là di se stessa, a differenza di un'opera tecnica che esiste per se stessa, possiede una funzione e un'utilità proprie e porta con sé la sua finalità ${ }^{36}$. Questi piccoli animali posti alla nostra attenzione da Quiet Ensemble portano con sé un carico di significati che rappresenta una possibilità di percepire mondi diversi dal nostro, che non sono legati a noi da vincoli di consumo o sfruttamento.

Già il nome scelto dal duo, questo insolito richiamarsi alla quiete per indicare la posizione che deve assumere lo spettatore, intriga. Lo vorremmo intendere in senso letterale, come "Ensemble quieto", grazie al significato in lingua inglese del vocabolo quiet, aggettivo che indica qualcosa di "tranquillo", "silenzioso", "quieto", e in senso spirituale, quando usato come nome, "modo di essere", o "stato di calma" e anche "segreto". Derivate tutte dalla parola latina quietus ${ }^{37}$, queste suggestioni suscitano alcune considerazioni che troviamo interessanti, e che prendiamo a prestito ancora dagli studi della filosofa tedesca ${ }^{38}$ :

\footnotetext{
${ }^{33}$ H. Arendt, Vita activa. La condizione umana, Bompiani, Milano 1958, p. 120.

${ }^{34}$ Ivi, p. 125.

${ }^{35}$ Cfr. O. Eliasson, op. cit., p. 17.

${ }^{36}$ J.L. Nancy, L'arte, oggi, in F. Ferrari (a cura di), Del contemporaneo. Saggi su arte e tempo, Bruno Mondadori, Milano 2007, p. 17.

${ }^{37}$ Definizioni del Longman Dictionary of Contemporary English, Pearson Education Ltd., Edinburgo 2000.

${ }^{38} \mathrm{H}$. Arendt (1906-1975) tra i maggiori filosofi del XX secolo, la Arendt, tedesca di origine ebraica, è emigrata in America per sfuggire al nazismo.
} 
Antica quanto Aristotele, la distinzione tra quiete e inquietudine, tra un'astensione quasi assoluta dal movimento fisico esterno e l'attività di ogni genere, è più decisiva che la distinzione tra il modo di vita politico e quello teoretico ${ }^{39}$. [...] Tutti i movimenti, quelli del corpo e dell'anima come quelli del linguaggio e della riflessione, debbono cessare di fronte alla verità. La verità, sia essa l'antica verità dell'Essere o la verità cristiana del Dio vivente, può rivelarsi soltanto in una completa serenità umana. ${ }^{40}$

Troviamo in queste considerazioni sulla necessità della quiete una chiave interessante per l'analisi dell'opera di Quiet Ensemble: nell'intento della factory di Vercelli e Di Salvo, allo spettatore "quieto" è proposta l'osservazione delle più piccole forme del vivente, colte nel lento svolgersi del loro movimento. Nel pensiero della Arendt, il silenzio del gesto si rivela paradossalmente come momento culmine dell'attività umana, suo rovescio necessario perché si raggiunga un efficace stato mentale che permetta la contemplazione del mondo come antidoto all'agitazione della vita attiva. Ugualmente, l'astensione dall'agire quotidiano proposta dai due artisti-performer, grazie alla creazione di uno spazio che richiede un'osservazione diversa, un'attenzione aumentata e quieta nei confronti della vita, consente l'emersione di un teatro del dettaglio che mette in scena il rivelarsi a noi dello spazio organico e naturale. E questo, secondo i diversi progetti realizzati, attraverso gli echi sonori che il vivente suscita o la cascata di punti luminosi che l'elettricità del movimento provoca.

\section{Esercitare la meraviglia}

Spiega Vercelli: «L'interesse era lavorare sulla lentezza e sulla pazienza: a prima vista sembrava non stesse succedendo nulla e quando lo spettatore ripassava dopo per esempio un'ora, poteva accorgersi che in realtà era cambiato tutto. ${ }^{41}$. In questo modo si ottiene la dilatazione della percezione relativa allo scorrere del tempo, dimostrando la parzialità della percezione quotidiana, spesso accelerata dal pensiero che continuamente sposta in avanti la nostra attenzione rispetto all'avvenimento presente.

\footnotetext{
${ }^{39} \mathrm{Cfr}$. Aristotele, Politica, 1333 a 30-33. Tommaso D’Aquino definisce la contemplazione come quies ab exterioribus motibus (Summa teologica, 2, 2, 179, 1). Cfr. H. Arendt, Vita activa. La condizione umana, Bompiani, Milano 1958, p. 244, nota 11.

40 Ivi, p. 12-13.

${ }^{41}$ B. Vercelli, op. cit.
} 
Nel prossimo progetto i due cercheranno di mettere in scena il fuoco. Li aspettiamo, per un'altra messinscena della natura all'interno di un percorso scenico che esplori la poesia del movimento minimo, proponendo performance che sono come una sorta di haiku visivi e sonori e che necessitano di tecniche di contemplazione "quieta" per poter essere compresi. Chiudo le mie riflessioni con le parole di Chandra Candiani: «La pratica della meraviglia è una pratica che cura anche il cuore più ferito della terra. Si può andare a trovare un piccolissimo pezzo di prato, un pizzico di prato c'è sempre, anche in città. $E$ guardare. A lungo. Si apre un universo minimo. Infinite vicende, mutamenti, arrivi, partenze, forme sempre più piccole man mano che lo sguardo si limita a vedere. Esercitare la meraviglia cura il cuore malato che ha potuto esercitare solo la paura» ${ }^{42}$.

\section{Riferimenti Bibliografici}

H. Arendt, Vita activa. La condizione umana, Bompiani, Milano 1958.

P. Auslander, Liveness, Performance in a mediatized culture, Routledge, London e New York 1999.

A. Balzola e A.M. Monteverdi, Le arti multimediali digitali. Storia, tecniche, linguaggi, etiche ed estetiche del nuovo millennio, Garzanti, Milano 2004.

K. Barad, Performatività della natura. Quanto e queer, Edizioni ETS, Pisa 2017.

J. Baudrillard, Pour une critique de l'économie politique des signes, Gallimard, Parigi 1972.

M. Borelli, N. Savarese, Te@tri nella rete. Arti e tecniche dello spettacolo nell'era dei nuovi media, Carocci, Roma 2004.

J.D. Bolter, R. Grusin, Remediation: Understanding New Media, MIT Press, Cambridge 1998.

J. Butler, A chi spetta una buona vita?, Nottetempo, Milano 2013.

C. Candiani, Questo immenso non sapere. Conversazioni con alberi, animali e il cuore umano, Einaudi, Torino 2021.

I. Caleo, Performance, materia, affetti. Una cartografia femminista, Bulzoni, Roma 2021.

O. Eliasson, Conversation entre Daniel Birnbaum et Olafur Eliasson, in M. Grynstejn, D. Birnbaum, M. Speaks (a cura di) Olafur Eliasson, Phaidon, London 2008.

\footnotetext{
${ }^{42}$ C. Candiani, Questo immenso non sapere. Conversazioni con alberi, animali e il cuore umano, Einaudi, Torino 2021, p.9.
} 
D.J. Haraway, Manifesto Cyborg, donne, tecnologie e biopolitiche del corpo, Feltrinelli, Milano 1995.

M. Kirby, On acting and not acting, in G. Battcock e R. Nickas (a cura di), The Art of Performance, Dutton, New York 1984.

J.L. Nancy, L'arte, oggi, in F. Ferrari (a cura di) Del contemporaneo. Saggi su arte e tempo, Bruno Mondadori, Milano 2007.

P. Phelan, Unmarked: The Politics of Performance, Routledge, London \& New York 1993.

A. Pizzo, Neodrammatico digitale. Scena multimediale e racconto interattivo, Accademia University Press, Torino 2013.

\section{Sitografia}

Olafur Eliasson: https://olafureliasson.net (ultimo accesso 28/11/2021), www.designboom.com/art/olafur-eliasson-interview-artist-designboom-02-16-2015 (ultimo accesso 28/11/2021).

Quiet Ensemble: https://www.quietensemble.com (ultimo accesso 28/11/2021), https://www.instagram.com/quiet_ensemble (ultimo accesso 28/11/2021).

Studio Rosengaard: www.studioroosegaarde.net/stories (ultimo accesso 28/11/2021).

\section{Recensioni internazionali a Quiet Ensemble}

CDM: https://cdm.link/2013/08/quiet-ensemble-music-by-pineapples-mice-snails-andgoldfish (ultimo accesso 28/11/2021).

CLOT: https://www.clotmag.com/interviews/quiet-ensemble-a-sympathetic-relationshipbetween-the-natural-and-unnatural (ultimo accesso 28/11/2021).

Creative Applications: https://www.creativeapplications.net/environment/sole-artificial-sunby-quiet-ensemble (ultimo accesso 28/11/2021).

Designboom: https://www.designboom.com/readers/quintetto-by-quiet-ensemble (ultimo accesso 28/11/2021).

Design Wanted: https://designwanted.com/architecture/quiet-ensemble-interview (ultimo accesso 28/11/2021).

Fast Company: https://www.fastcompany.com/1669473/what-fruit-sounds-like-when-youturn-its-natural-electricity-into-techno-beats (ultimo accesso 28/11/2021).

FRAME: https://www.frameweb.com/article/digital-music-by-quiet-ensemble (ultimo accesso $28 / 11 / 2021)$. 
Huffington Post: https://www.huffpost.com/entry/biografie-aims-to-capture_n_1373915; https://www.huffpost.com/entry/natura-morta_n_1413176 (ultimo accesso 28/11/2021).

Laughing Squid: https://laughingsquid.com/an-orchestra-of-lights-creates-eerie-sounds-in-anew-performance-installation-by-quiet-ensemble (ultimo accesso 28/11/2021).

Le Devoir: https://www.ledevoir.com/culture/actualites-culturelles/492496/ne-rien-faire-aI-affiche (ultimo accesso 28/11/2021).

Lightecture: https://www.lightecture.com/arte_digital/sole-los-rayos-de-un-sol-artificial (ultimo accesso 28/11/2021).

StirWorld: https://www.stirworld.com/see-features-quiet-ensemble-explores-the-naturaland-the-artificial-in-technology-through-design (ultimo accesso 28/11/2021).

Vice: https://www.vice.com/en/article/kbn7gv/strobing-lights-are-the-instruments-in-thequiet-ensembles-robotic-symphony (ultimo accesso 28/11/2021).

Wired: https://www.wired.com/2014/12/trippy-orchestra-made-entirely-lamps-electricity (ultimo accesso 28/11/2021).

XIUMI: https://b.xiumi.us/stage/v5/3ZQUV/253382416 (ultimo accesso 28/11/2021).

\section{Recensioni italiane a Quiet Ensemble}

Artwort: https://www.artwort.com/2020/10/21/arte/digital-art/sole-quiet-ensemble (ultimo accesso 28/11/2021).

Exibart: https://www.exibart.com/progetti-e-iniziative/unshaped-quiet-ensemble-palermo (ultimo accesso 28/11/2021).

InsideArt: http://insideart.eu/2018/07/11/intervista-con-quiet-ensemble (ultimo accesso 28/11/2021).

Internazionale: https://www.internazionale.it/video/2015/01/22/suonare-con-la-luce (ultimo accesso 28/11/2021).

Rockit: https://www.rockit.it/intervista/quiet-ensemble-enlightenment-musica-luci (ultimo accesso 28/11/2021).

Segno: https://segnonline.it/quiet-ensemble-primitive-primavere-alla-sala-santa-rita (ultimo accesso 28/11/2021).

Vice: https://www.vice.com/it/article/rbny85/quiet-ensemble-interview (ultimo accesso 28/11/2021). 


\section{Biografia dell'autore/ Author's biography}

Maia Giacobbe Borelli, studiosa e insegnante, si occupa di nuovi media nel campo dello spettacolo e delle sue implicazioni sociali. Dopo una lunga esperienza come consulente audiovisiva per la Commissione europea a Roma (1994-2003) e ancora prima come produttore di video e documentari con la società Tape Connection da lei fondata e diretta (1984-1993), ha collaborato con la Sapienza Università di Roma, dove ha ottenuto nel 2008 un Dottorato di ricerca in co-tutela di tesi con I'Università Paris 7, cattedra di Ethnologie Visuelle, relativo ai testi dell'ultimo Antonin Artaud (i Cahiers di Rodez). Cultore della materia per la cattedra di Storia del Teatro e dello Spettacolo, ha partecipato per il Centro Teatro Ateneo alla messa in rete del patrimonio audiovisivo europeo di spettacolo (Progetto ECLAP 2010-2013). Ha pubblicato per i tipi di Carocci (2004), Dino Audino (2011), Bulzoni (2012), Springer (2013), Editoria \& Spettacolo (2015) e per le riviste Alfabeta, Ariel, Mimesis Journal, Biblioteca teatrale, Antropologia e Teatro, Teatri della Diversità. Fa parte di ORMETE (oralità, memoria, teatro) per il progetto di ricerca sul teatro curato da D. Orecchia e L. Cavaglieri con le Università di Roma, Genova e Parma.

Teacher and researcher in Performing Arts and their social implications face to New Media. In 2008 she obtained a PhD co-directed by the Université Paris 7 and the Departement of Digital Technologies for the Performing Arts (Sapienza University of Rome). She is a specialist of the latest corpus of Antonin Artaud's work. From 2010 to 2013 she collaborated with Centro Teatro Ateneo, Sapienza University of Rome, the research centre on Performing Arts for the ECLAP, European Content and Learning Arts of Performing digital archive. She published with Carocci (2004), Dino Audino (2011), Bulzoni (2012), Springer (2013), Editoria \& Spettacolo (2015) and for several academic journals and specialized magazines as Alfabeta, Ariel, Mimesis Journal, Biblioteca teatrale, Antropologia e Teatro, Teatri della Diversità. Main publishing: Out of Order. Quel che resta del corpo nello spettacolo contemporaneo (ed.) Bulzoni, Roma 2012; II teatro e la Rete: dal corpo al "senza corpo" nella nuova scena digitale in "La scena tecnologica. Dal video in scena al teatro interattivo", (ed. by Andrea Balzola), Dino Audino, Roma 2011; (with Nicola Savarese) Te@tri nella Rete, arti e tecniche dello spettacolo nell'era dei nuovi media, Carocci, Roma 2004. Actually working with ORMETE (orality, memories and theatre) a research project led by D. Orecchia and L. Cavaglieri with the University of Rome, Genoa and Parma.

Articolo sottoposto a double-blind peer review 